\title{
Sorting of Acrylonitrile-Butadiene-Styrene and Polystyrene Plastics by Microwave Cavity Resonance
}

\author{
Yuya Mori $^{\mathrm{a}}$, Takehiko Kobayashi ${ }^{\mathrm{a}}$, and KenTahara ${ }^{\mathrm{b}}$ \\ ${ }^{a}$ Wireless Systems Laboratorory, Tokyo Denki University, 5 Senju-Asahi-Cho, Tokyo, 120-8551 Japan \\ ${ }^{b}$ Kanto Electronic Application and Development Corporation, Tokyo, 186-0011, Japan
}

\begin{abstract}
This paper presents a new sorting method for acrylonitrile-butadiene-stryrene (ABS) and poly styrene (PS) by means of microwave cavity resonator perturbation. In this method, a small piece of sample material is inserted into a cavity resonator at a point when the electric and magnetic fields mark the maximum and the minimum values, respectively. Experiments were carried out with use of 1-, 2-, 5-, and 10-GHz resonators and ABS and PS samples of various sizes. Loss angles of polarizability $(\tan \chi)$ exhibited differences large enough to achieve accurate sorting between ABS and PS.
\end{abstract}

Index Terms: Cavity resonator, Microwave measurement.

(C) 2016 Published by MECS Publisher. Selection and/or peer review under responsibility of the Research Association of Modern Education and Computer Science

\section{Introduction}

The amount of waste electric and electronic equipment is increasing constantly, driven by the ever-growing use of technology. Plastic recycling has been attracting great attention due to its ecological and economic impacts [1]. Though acrylonitrile-butadiene- styrene (ABS) and polystyrene (PS) are widely used in household and office appliances, sorting of these two resins is particularly challenging, since ABS and PS are almost identical in appearance. A conventional selecting method utilizes sink-float separation [2], based on the difference in specific gravities. However, specific gravities of ABS and PS partially overlap. Another method is electrostatic selecting, which utilizes the difference in charging rate [3]. This method employs an electrostatic field which exerts a force on frictionally charged plastics and utilizes a difference in travel distances for selection. However, since the ABS and PS resins are very close in the triboelectric series, differences of surface conditions may reverse their electrification polarity. The third method is infrared spectroscopy, based on the difference in infrared spectra reflected from the sample. This method can sort the two resins more accurately

* Corresponding author.

E-mail address: yuya@grace.c.dendai.ac.jp 
than the previous two, but cannot be used for black- or dark-colored samples that absorb infrared energy [4].

\section{Dielectric Dispersion of ABS and PS}

Real and imaginary parts of complex permittivity $\left(\varepsilon\right.$ and $\left.\varepsilon^{\prime \prime}\right)$ of typical polar dielectrics are dispersed over an electromagnetic spectrum. Within the spectrum, orientation polarization is dominant in the microwave frequencies, and the orientation polarization is generated by permanent dipole moment of polar groups, such as hydroxyl, carboxyl, and nitrile groups. Polar molecules, such as water, ethyl alcohol, and hydrogen chloride, exhibit large $\varepsilon$ and $\varepsilon$ ", while non-polar molecules, such as methane, carbon dioxide, and chloride, exhibit small $\varepsilon$ and $\varepsilon$ ". The ABS is a terpolymer produced by polymerizing styrene and acrylonitrile in the presence of polybutadiene. The proportions can vary from 25 to $30 \%$ acrylonitrile, 25 to $30 \%$ butadiene, and 40 to $50 \%$ styrene. Of the three components, acrylonitrile has a nitrile radical possessing strong polarity. Therefore, ABS is classified into polar plastics. On the other hand, PS is nonpolar, since it is produced by polymerizing styrene, containing only hydrogen and carbon.

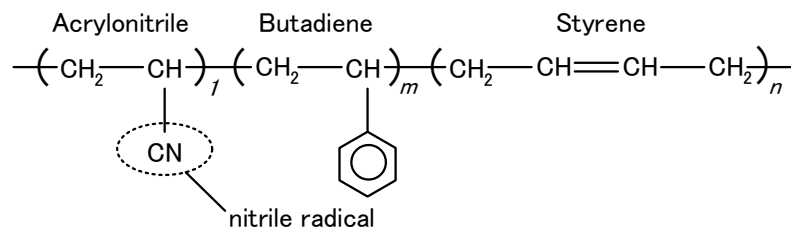

(a)

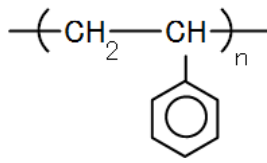

(b)

Fig.1. Chemical formula of (a) ABS; (b) PS

\section{Proposed Method}

We proposed a method for sorting ABS and PS by means of microwave cavity resonator perturbation, which is commonly used for evaluating microwave complex permittivity [5]. Figure 2 depicts a measurement system of cavity resonance perturbation method with use of an either vector or scaler microwave network analyzer. When a resonant cavity is perturbed, e.g. when a dielectric is inserted into the cavity, electromagnetic fields inside the cavity change accordingly. If the volume of the dielectric is small compared to that of the cavity, the electromagnetic fields after the change differ by a very small amount from the fields before the change, and a perturbation theory can be applied. The dielectric sample is usually inserted into the cavity at a point when the electric and magnetic fields mark the maximum and the minimum values, respectively. Shifts in resonant frequency and the quality factor (Fig. 3) are given by complex permittivity of the dielectric [6] :

$$
\varepsilon^{\prime}=1+\frac{V}{\alpha \nu}\left(1-\frac{f_{s}}{f_{0}}\right)
$$

and 


$$
\varepsilon^{\prime \prime}=\frac{V}{2 \alpha \nu}\left(\frac{1}{Q_{s}}-\frac{1}{Q_{0}}\right)
$$

where

$V: \quad$ volume of the cavity

$v: \quad$ volume of the sample

$f_{0}: \quad$ resonant frequency of the empty cavity

$f_{s}: \quad$ resonant frequency after insertion of the sample into the cavity

$Q_{0}: \quad$ quality factor of the empty cavity

$Q_{s}: \quad$ quality factor after insertion of the sample into the cavity

$\alpha: \quad$ proportionality constant.

Microwave network analyzer

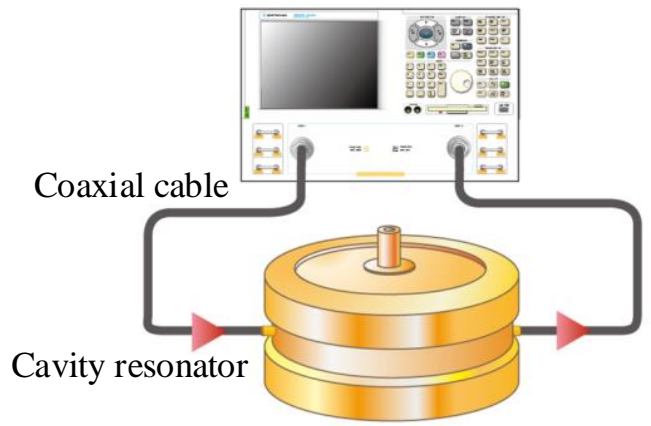

Fig.2. A measurement system of the cavity resonance perturbation method.

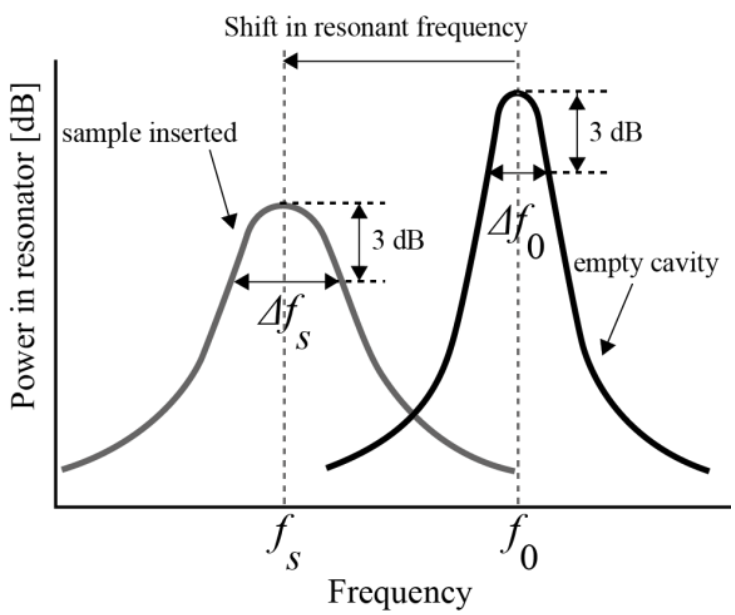

Fig.3. Resonance characteristics. When the dielectric sample is inserted into the cavity, the resonance frequency shifts from $f_{0}$ to $f_{s}$ and the quality factor from $Q_{0}=f_{0} / \Delta f_{0}$ to $Q_{s}=f_{s} / \Delta f_{s}$. 
The sample volume $v$ is difficult to measure accurately and the proportionality constant $\alpha$ depends on the resonance mode $\left(\mathrm{TM}_{010}: 1.855, \mathrm{TM}_{020}: 4.318\right)$. Instead of $\varepsilon$ ' and $\varepsilon "$, we proposed a use of loss angle of polarizability, $\tan \chi[5]$, defined by

$$
\tan \chi=\frac{\varepsilon^{\prime \prime}}{\varepsilon^{\prime}-1}=\frac{f_{0}}{2\left(f_{0}-f_{s}\right)}\left(\frac{1}{Q_{s}}-\frac{1}{Q_{0}}\right),
$$

which contains only the shifts in the resonant frequency and the quality factor, excluding $V, v$, and $\alpha$.

\section{Measurement Apparatus}

\subsection{Structure}

Four microwave cylindrical cavity resonators were used, as shown in Fig. 4, and the unloaded quality factors and the resonant modes are listed in Table 1 . The cutout view of these cylindrical cavity resonators are shown in Fig. 5. Each resonator had an insertion hole at its center. Each hole had a polytetrafluoroethylene (PTFE) sleeve of $2.7 \mathrm{~mm}$ inner diameter. This sleeve is used to secure the sample at the position of the maximum electric field.

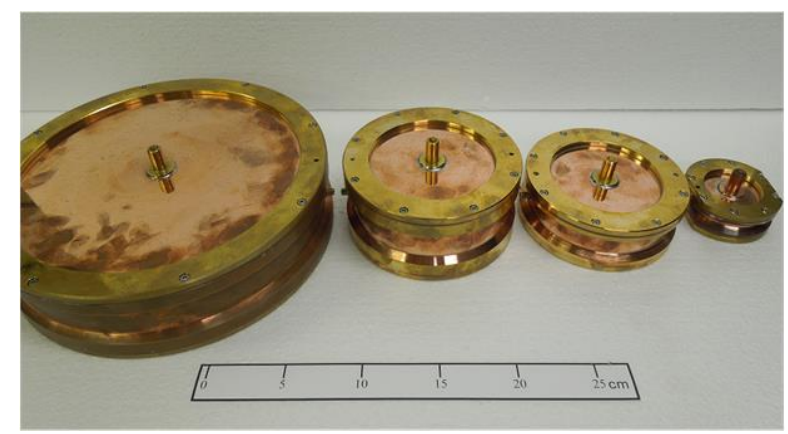

Fig.4. $\mathrm{TM}_{0 \mathrm{~m} 0}$ mode cylindrical cavity resonators. From left to right, $1,2,5,10 \mathrm{GHz}$ resonators.

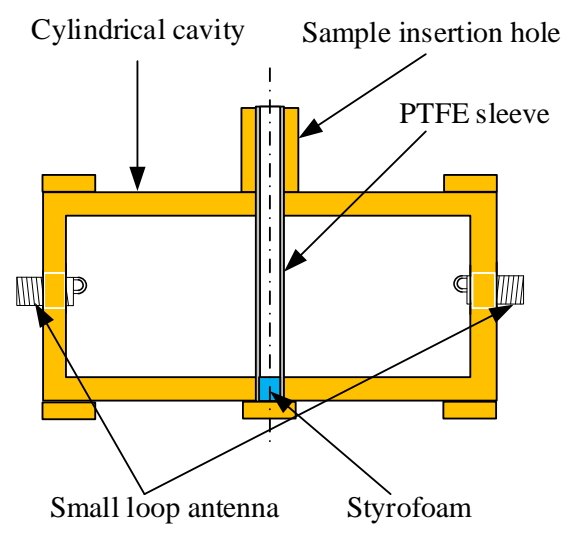

Fig.5. Structure of cylindrical cavity. 
Table 1 . Unloaded quality factor (at $25 \pm 3^{\circ \mathrm{C}}$ )

\begin{tabular}{lll}
\hline Resonant frequency & Quality factor & Resonant mode \\
\hline $1 \mathrm{GHz}$ & $15300 \pm 100$ & $\mathrm{TM}_{010}$ \\
$2 \mathrm{GHz}$ & $14800 \pm 100$ & $\mathrm{TM}_{010}$ \\
$5 \mathrm{GHz}$ & $16800 \pm 100$ & $\mathrm{TM}_{020}$ \\
$10 \mathrm{GHz}$ & $8300 \pm 100$ & $\mathrm{TM}_{020}$ \\
\hline
\end{tabular}

\subsection{Measurement Apparatuses}

We prepared ABS and PS samples of three shapes: namely, (A) long square cylinder, (B) shorter square cylinder, and (C) plural granular. The long square cylinder samples (A) of ABS (uncolored, black, and white) and PS (white and black) were prepared in a size of approximately $1 \mathrm{~mm} \times 1 \mathrm{~mm} \times 80 \mathrm{~mm}$ (longer than all resonator heights), as shown in Fig. 6. These samples were cut out from a 1-mm thick sheets. Variances of the cross sections were $(1.00 \pm 0.02) \mathrm{mm} \times(1.02 \pm 0.01) \mathrm{mm}$. Shorter samples $(B)$ were also parallelepipeds whose cross section was $(1.00 \pm 0.02) \mathrm{mm} \times(1.02 \pm 0.01) \mathrm{mm}$, as shown in Fig. 7. The length of the samples were 1, 5, 10, 15, and $20 \mathrm{~mm}$. The numbers after ABS and PS represent the length of the sample: namely 1, 5, 10, 15, and $20 \mathrm{~mm}$. All the samples were white. When measuring the shorter samples, the samples were placed below the bottom of the resonator. Thereby, the displacement current which flowed into a sample from the surface of a wall increased, and the resonator loss also increased. Plural granular samples (C) were chopped approximately 0.5 to $3.0 \mathrm{~mm}$ long from $1 \mathrm{~mm} \times 1 \mathrm{~mm} \mathrm{ABS}$ and PS (white) square cylinders, as shown in Fig. 8 .

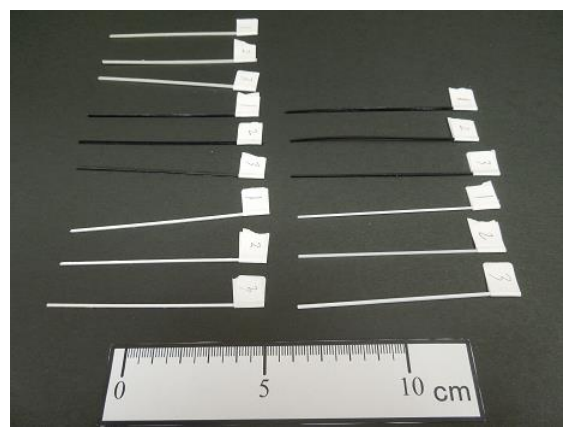

Fig.6. Square cylinder sample (A) of ABS and PS

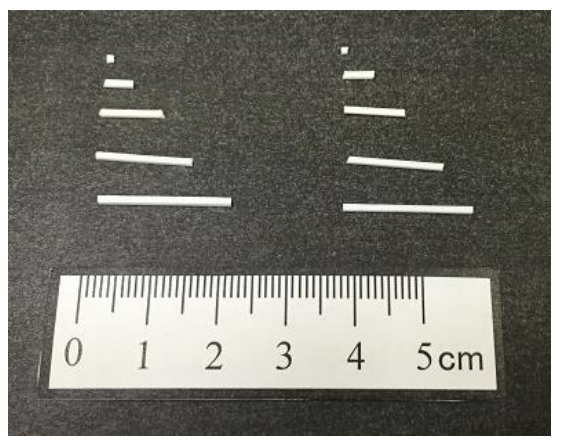

Fig.7. Shorter sample (B) of ABS and PS. 


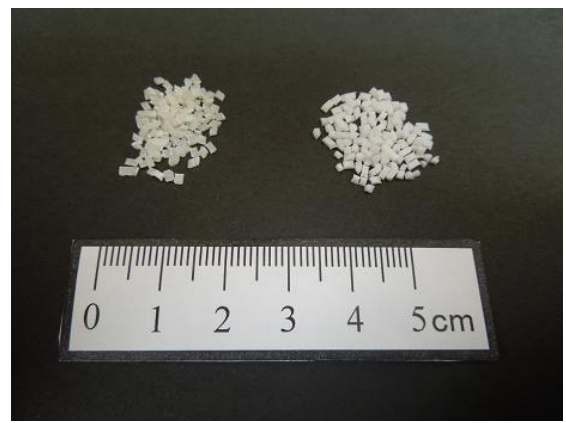

Fig.8. Plural granular samples (C) of ABS and PS.

\section{Measurement Results}

The longer samples (A) of ABS and PS, uncolored and black- and white-colored, were measured by using the four resonators, and $\tan \chi$ values were derived therefrom, as shown in Fig. 9. Each plot represents the average and the standard deviation of 50-time measurements (using three samples). A clear separation between ABS and PS was found at the four frequencies, nearly independent of sample colors, although the blackcolored samples tended to yield longer $\tan \chi$ than the uncolored or white-colored, as shown by solid symbols in Fig. 9.

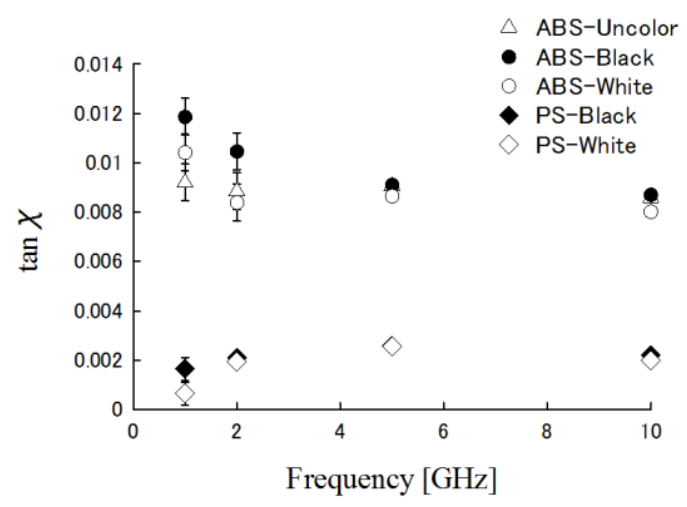

Fig.9. Measured $\tan \chi$ of samples longer than the resonator height (A).

Figure 10 shows the results using white-colored samples of length shorter than or equal to resonator height (B) at $10 \mathrm{GHz}$. When using less than $5 \mathrm{GHz}$ resonators, change in the resonance characteristics could not be confirmed from the samples, which were too small compared to the cavity volume. Though $f_{s}$ and $Q_{s}$ depended on the sample length, a clean separation in $\tan \chi$ between ABS and PS was again observed, as shown Fig. 10. 


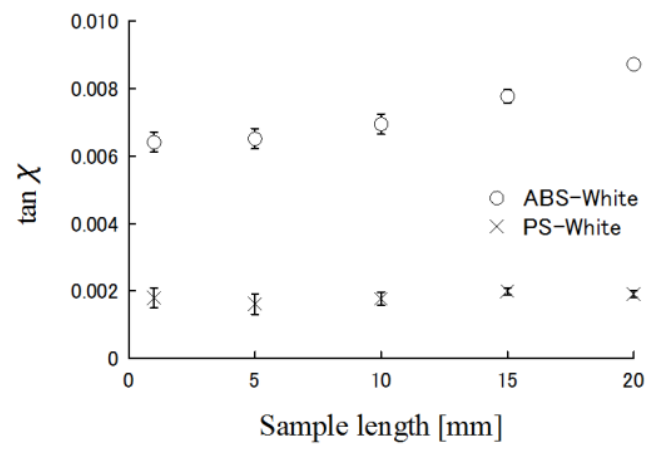

Fig.10. Measured $\tan \chi$ of samples shorter than or equal to the resonator height (B) at $10 \mathrm{GHz}$.

Figure 11 presents the measurement results using plural granular samples (C) at $1 \mathrm{GHz}$. Since the $1 \mathrm{GHz}$ resonator was the biggest between the four, it was most suited to the experiment. Figures 11 (a) and (b) show the shifts in the resonant frequency and quality factor, respectively. Figure 11 (c) again depicts clean separations in $\tan \chi$ between ABS and PS, almost independently of the total volume of the sample.

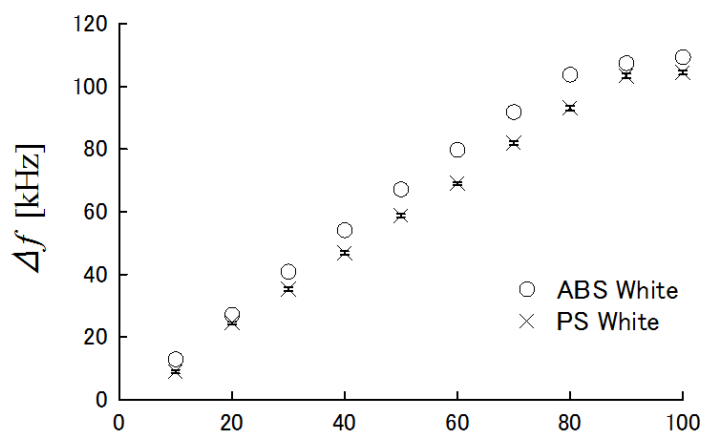

Total volume of the granular samples $\left[\mathrm{mm}^{3}\right]$

(a)

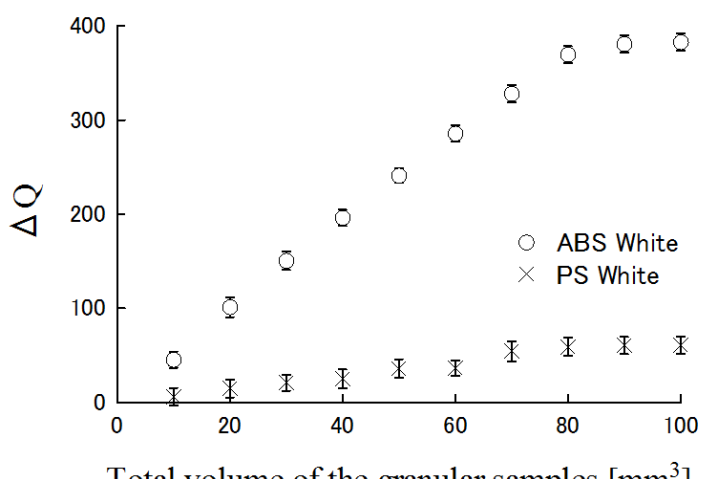

(b) 


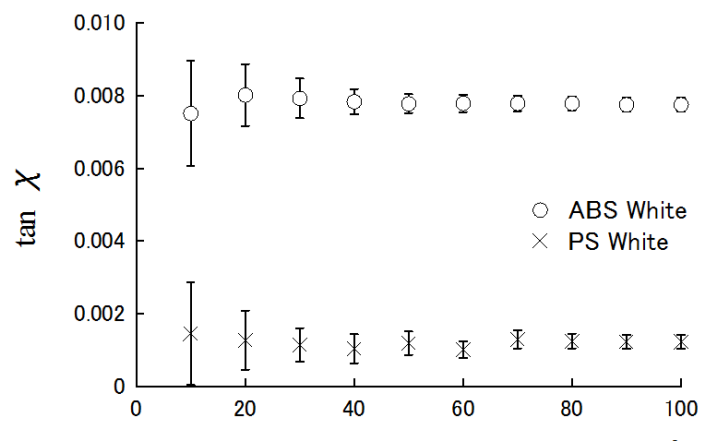

Total volume of the granular samples $\left[\mathrm{mm}^{3}\right]$

(c)

Fig.11. Measured $\tan \chi$ of granular samples (C) height at $1 \mathrm{GHz}$ : (a) Shift in the resonant frequency, (b) Shift in the quality factor, and (c) $\tan \chi$.

\section{Conclusions}

This paper presents a new method for sorting ABS and PS based on microwave cavity resonator perturbation. Shifts in resonant frequency and quality factors were measured with a vector network analyzer when a small sample was inserted into a resonant cavity. Clear separation between ABS and PS was observed with use of tan $\chi$. Results were almost independent of colors and found approximately constant regardless of coloration, shape (e.g. long square cylinder and granular) and volume of the sample. Small samples (even $1 \times 1 \times 1 \mathrm{~mm}^{3}$ ) can be classified with this method.

\section{References}

[1] F. O. Ongondo, I. D. Williams, and T. J. Cherrett, "How are WEEE doing? A global review of the management of electrical and electronic wastes," Waste Management, 31, pp. 714-730, 2011.

[2] N. Fraunholcz, "Separation of waste plastics by froth flotation-a review, part 1," Minerals Engineering, 17, pp. 261-268, 2004.

[3] K. Haga, "Applications of the electrostatic separation technique", in J. S. Chang, A. J. Kelly, and J. M. Crowley (Eds), Handbook of Electrostatic Processes, Marcel Dekker, NewYork, 1995.

[4] G. Bonifazi, F. Di. Maio, F. Potenza, S. Serranti, "FT-IR spectroscopy and hyperspectral imaging applied to post-consumer plastic packaging characterization and sorting," IEEE SENSORS 2014, pp. 633-636, Valencia, Spain, Nov. 2-5, 2014.

[5] Y. Mori, T. Kobayashi and K. Tahara, "Sorting of Acrylonitrile-Butadiene-Styrene and Polystyrene Plastics by Microwave Cavity Resonance," IEEE Radio and Antenna Days of the Indian Ocean (IEEE RADIO2015), Belle Mare, Mauritius, Sep. 21-24, 2015.

[6] H. Bethe and J. Schwinger, "Perturbation theory for cavities," NDRC Report Cornell University, D1-117, 1943. 


\section{Authors' Profiles}

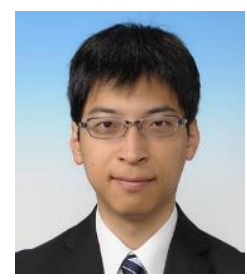

Yuya Mori received the B.E. degrees from the Tokyo Denki University, Tokyo, Japan in 2014. He is now pursing a M.E. degree from Tokyo Denki University, Tokyo, Japan. His research interests include measurement of material constants in microwave.

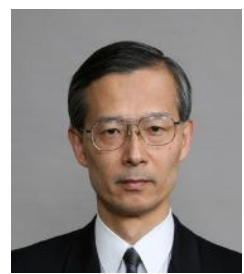

Takehiko Kobayashi received the B.E., M.E., and Ph.D. degrees in electrical engineering from the University of Tokyo in 1978, 1980, and 1983. He joined Nippon Telegraph and Telephone in 1983 and was engaged in research on various wireless communication systems. He was guest scientist at National Bureau of Standard (now NIST) in Boulder, Colorado in 1986. From 1998 to 2001, he was with YRP Key Tech Labs, which focuses on the 4th generation mobile communication systems. Currently, he is a professor at the Department of Information and Communication Engineering, Tokyo Denki University. He received the IEICE Best Paper Awards in 2001 and 2002, and the Telecom System Awards from the Telecommunications Advancement Foundation in 2003 and 2005. His current research interests include ultra wideband wireless systems, mobile communication channel characterization, and teletraffic evaluation of mobile communication networks.

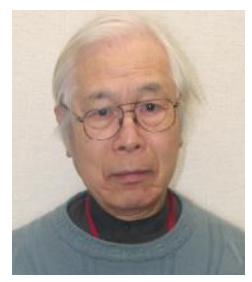

Ken Tahara received the B.E. degree in electronic engineering from Kogakuin University, Tokyo, Japan in 1964. He had been working at a laboratory of Nippon Telegraph and Telephone since 1958 and then founded Kanto Electronic Application and Development Corporation in 1972, focusing on microwave and millimeter wave technologies, particularly their application to radio astronomy.

How to cite this paper: Yuya Mori, Takehiko Kobayashi, KenTahara,"Sorting of Acrylonitrile-ButadieneStyrene and Polystyrene Plastics by Microwave Cavity Resonance", International Journal of Wireless and Microwave Technologies(IJWMT), Vol.6, No.2, pp.1-9, 2016.DOI: 10.5815/ijwmt.2016.02.01 